\title{
TECNOLOGIAS DA INFORMAÇÃO E COMUNICAÇÃO NAS INSTITUIÇÕES DE ENSINO SUPERIOR: INTERFACES COM O CAMPO DA SAÚDE
}

\author{
INFORMATION AND COMMUNICATION TECHNOLOGIES IN HIGHER \\ EDUCATION INSTITUTIONS: INTERFACES WITH THE HEALTH \\ FIELD
}

Amauri dos Santos Araujo ${ }^{1}$ Izadora Nunes da Silva ${ }^{2}$ Glaucia dos Santos Silva ${ }^{3}$ Vanessa Maria do Nascimento ${ }^{4}$

\begin{abstract}
RESUMO: O presente estudo alude à necessidade de identificar o impacto das Tecnologias da Informação e Comunicação (TICs) nas instituições de ensino superior. OBJETIVO: Identificar o impacto no uso das Tecnologias da Informação e Comunicação na formação em Enfermagem nas instituições de ensino superior no Brasil. MÉTODOS: Trata-se de um estudo de revisão integrativa, realizado nas bases de dados da BVS, MEDLINE, LILACS e BDENF, a partir do recorte temporal de estudos publicados entre 2013 a 2018. RESULTADOS E DISCUSSÃO: Foram localizados 05 artigos, apresentando deste modo, variações acerca do processo da educação à distância. Destarte, o cenário de desenvolvimento e acumulo de conhecimento, tem sido ponto de destaque nos estudos, reforçando que as condições causais, inerentes, intervenientes e consequências relacionadas às Tecnologias da Informação e Comunicação. CONCLUSÃO: Observa-se nos estudos um amplo aspecto acerca dos benefícios e riscos do ensino em enfermagem por meio do uso de plataformas e interação colaborativa por WebQuest suprindo lacunas na formação. Contudo, destaca-se que o melhor comportamento para este tipo de formação está atrelado a educação permanente e continuada dos profissionais, e em nível de especialização.
\end{abstract}

Palavras chave: Educação. Enfermagem. Saúde. Tecnologia.

\footnotetext{
${ }^{1}$ Enfermeiro. Mestrando em enfermagem pelo PPGENF/EENF/UFAL. Professor da Faculdade de Tecnologia de Alagoas. E-mail: amauriaraujo.sms@gmail.com.

${ }^{2}$ Graduanda em Enfermagem pela Faculdade de Tecnologia de Alagoas.

${ }^{3}$ Graduanda em Enfermagem pela Faculdade de Tecnologia de Alagoas.

${ }^{4}$ Graduanda em Enfermagem pela Faculdade de Tecnologia de Alagoas.
} 
ABSTRACT: The present study alludes to the need to identify the impact of information and Communication Technologies (ICTs) in higher education institutions. OBJECTIVE: To identify the impact on the use of Information and Communication Technologies in nursing education at higher education institutions in Brazil. METHOD: This is an integrative review study, carried out on the basis of data from the VHL, MEDLINE, LILACS and BDENF, from the time frame of studies published between 2013 and 2018. RESULTS AND DISCUSSION: We found 05 articles, presenting in this way, variations on the process of distance education. Thus, the scenario of development and accumulation of knowledge, has been point of studies, stressing that the causal, inherent, intervening and consequences related to Information and Communication Technologies. CONCLUSION: In the studies there is a broad aspect about the benefits and risks of teaching in through the use of platforms and collaborative interaction by WebQuest filling gaps in training. However, it is emphasized that the best behavior forThis type of training is linked to the continuing and continuing education ofprofessionals, and at the level of specialization.

Keywords: Education. Nursing. Health. Technology. 


\section{INTRODUÇÃO}

O presente estudo alude à necessidade de identificar o impacto das Tecnologias da Informação e Comunicação (TICs) nas instituições de ensino superior. Com esta percepção, nota-se que as transformações que ocorrem devido ao surgimento e fortalecimento das TICs, ultrapassam as fronteiras e especificidades e, se mostram como ferramenta imprescindível no desenvolvimento das relações interpessoais (PRADO et al., 2012).

Nesta perspectiva, o Brasil, através da Lei de Diretrizes e Bases da Educação Nacional (LDB), está em consonância com os princípios da declaração mundial sobre a educação superior. Deste modo, proporciona um cenário autônomo e com liberdade acadêmica desafiando o processo educacional e os educadores a utilizarem novas tecnologias (LEI DE DIRETRIZES E BASE EDUCACIONAL, 1996).

Nesse processo de inserção das tecnologias, o computador sem dúvida, tem sido o mais utilizado pela sociedade e que por ser considerado como um ícone máximo na tecnologia e repercute com ágil comunicação. Com os avanços surgem profundas mudanças sobre educação superior. A utilização de sistemas de informação que auxilie para a tomada de decisão da enfermagem surge com um alicerce para o salto na qualidade da assistência, gerência, ensino e pesquisa, tornando cada fase do processo de enfermagem interativa e dinâmica no âmbito da formação profissional (PRADO et al., 2012; MOREIRA et al., 2013).

Diante do exposto, emerge a seguinte questão norteadora para o estudo: quais as possibilidades e limitações do uso das Tecnologias da Informação e Comunicação no ensino superior? Deste modo, emerge o seguinte objetivo do estudo: Identificar o impacto no uso das Tecnologias da Informação e Comunicação na formação em Enfermagem nas instituições de ensino superior no Brasil. 


\section{MÉTODOLOGIA}

Trata-se de um estudo de revisão integrativa; ao qual permite a síntese de trabalhos para a realização de uma ampla abordagem metodológica referente a revisões, partindo da inclusão de estudos experimentais e não experimentais para uma compreensão completa do fenômeno analisado (WHITTEMORE; KNAFL, 2005; ARAUJO et al., 2018).

Para este tipo de estudo, faz-se necessário percorrer seis etapas distintas: identificação do tema e seleção da hipótese ou questão de pesquisa; estabelecimento de critérios para inclusão e exclusão de estudos/amostragem ou busca na literatura; definição das informações a serem extraídas dos estudos selecionados/ categorização dos estudos; avaliação dos estudos incluídos na revisão integrativa; interpretação dos resultados; apresentação da revisão/síntese do conhecimento (WHITTEMORE; KNAFL, 2005; ARAUJO et al., 2018).

Para o embasamento teórico, foi realizada uma busca a partir das bases de dados Literatura Internacional em Ciências da Saúde (MEDLINE), Literatura LatinoAmericano e do Caribe em Ciência da Saúde (LILACS) e Base de Dados de Enfermagem (BDENF), utilizando-se os seguintes Descritores em Ciência da Saúde (DeCS): "tecnologia", "saúde", "enfermagem" e "ensino superior". Como estratégia de busca foi realizando o cruzamento dos DeCS com o booleano AND, conforme demonstra Tabela 1.

Como critérios de inclusão foi realizado o recorte temporal de estudos publicados entre 2013 a 2018, estudos nacionais publicados no idioma português, com disponibilidade de texto na íntegra, e como caraterísticas central, que um dos autores seja profissional enfermeiro. Foram excluídos estudos internacionais, trabalhos de conclusão de curso, monografias e teses, e estudos que não se relacionam no contexto da educação para a área da saúde. 
Tabela 1 - Processo de seleção dos artigos após leitura integral do estudo - Brasil, 2019.

\begin{tabular}{ccccc}
\hline DeCS & $\begin{array}{c}\text { Base de } \\
\text { Dados }\end{array}$ & $\begin{array}{c}\text { Artigos } \\
\text { Encontrados }\end{array}$ & $\begin{array}{c}\text { Artigos } \\
\text { Excluídos }\end{array}$ & Selecionados \\
\hline \multirow{2}{*}{ Tecnologia AND Saúde } & MEDILINE & $\mathbf{n}$ & $\mathbf{n}$ & $\mathbf{n}$ \\
AND Enfermagem AND & LILACS & 10 & 01 & - \\
Educação & BDENF & 08 & 08 & 02 \\
& TOTAL & $\mathbf{1 9}$ & 05 & 03 \\
& & & $\mathbf{1 3}$ & $\mathbf{0 5}$
\end{tabular}

\section{RESULTADOS E DISCUSSÃO}

Foram localizados 19 estudos a partir do cruzamento de dados, conforme apresenta a Tabela 1. Após refinamento conforme critérios de inclusão estabelecidos foram selecionados 05 artigos, conforme descreve o Quadro 2, traçando perfis variáveis dentro do contexto educacional. Destarte, referenciam-se em maior qualificação os níveis de educação permanente e continuada e em nível de especialização, em uso de TICs por meio de plataformas, WebQuest e atividades de desenvolvimento interpessoal e relacional. 
Quadro 1 - Síntese dos estudos de produção científica sobre o uso das TICs na formação em Enfermagem no Ensino Superior. Brasil, 2019.

\begin{tabular}{|c|c|c|c|c|}
\hline \multicolumn{2}{|c|}{ Título do Artigo } & Autores/Ano & Métodos & Resultados/Conclusões \\
\hline$P 1$ & $\begin{array}{|lr|}\text { Educação } & \\
\text { permanen } & \text { te: } \\
\text { Tecnologi } & \text { a } \\
\text { para } & \text { al } \\
\text { prevenção } & \text { do } \\
\text { erro } & \text { de } \\
\text { medicação } & \\
\end{array}$ & $\begin{array}{l}\text { VILELA, } \\
\text { R.P.B. et al. } \\
(2017)\end{array}$ & $\begin{array}{l}\text { Estudo } \\
\text { descritivo- } \\
\text { exploratório, } \\
\text { documental, } \\
\text { retrospectiv o. }\end{array}$ & $\begin{array}{l}\text { As oportunidade de treinamento relacionados } \\
\text { à prevenção do erro de medicação e com } \\
\text { temas diversificados, evidencia a a } \\
\text { necessidade em estratégias visando } \\
\text { aumentar a média de participação dos } \\
\text { profissionais de enfermagem no processo de } \\
\text { educação. }\end{array}$ \\
\hline$P 2$ & 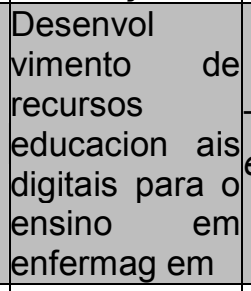 & TIBES, C.M., & $\begin{array}{l}\text { Pesquisa } \\
\text {,explicativa na } \\
\text { metodologia de } \\
\text { WebQuest. }\end{array}$ & $\begin{array}{l}\text { Na avaliação de clareza e pertinência os } \\
\text { cursos e conteúdos desenvolvidos foram } \\
\text { considerados adequados. Entretanto, para } \\
\text { que as WebQuest desenvolvidas sejam } \\
\text { amplamente disponibilizadas, ainda é } \\
\text { necessário que haja mais avaliações quanto } \\
\text { à sua qualidade técnica e usabilidade. }\end{array}$ \\
\hline$P 3$ & \begin{tabular}{|lr} 
Acidenter & de \\
trabalho & com \\
material & \\
biológico & no \\
contexto & de \\
um ambiente \\
virtual r de \\
aprendizagem
\end{tabular} & $\begin{array}{l}\text { FREGUIA, } \\
\text { A.F.S., et al. } \\
(2016) \text {. }\end{array}$ & $\begin{array}{l}\text { Uso } \quad \text { de } \\
\text { WebQuest em } \\
\text { 1. plataforma } \\
\text { educacional } \\
\text { Moodle. }\end{array}$ & $\begin{array}{l}\text { Confirmou-se a importância das TICs como } \\
\text { instrumento para a prática docente } \\
\text { mediadora, na integração r entre } \\
\text { conhecimentos válidos e a realidade } \\
\text { complexa e dinâmica dos serviços de saúde. }\end{array}$ \\
\hline P4 & $\begin{array}{|lr|}\text { Vivencian do o } \\
\text { uso } & \text { das } \\
\text { Tecnologi as } \\
\text { da } r \text { Informaçã } \\
\text { o e } & \text { Comunica } \\
\text { ção e } & \text { e suas } \\
\text { dificuldad es } \\
\text { no processo } \\
\text { ensino- } \\
\text { aprendizagem }\end{array}$ & $\begin{array}{l}\text { LEITE, } \\
\text { K.N.S., } 2014 .\end{array}$ & $\begin{array}{l}\text { Estudo } \\
\text { exploratório } \\
\text { qualitativo }\end{array}$ & $\begin{array}{l}\text { As condições causais, inerentes, } \\
\text { intervenientes e consequências relacionadas } \\
\text { às Tecnologias da Informação e } \\
\text { Comunicação (TIC) no processo de ensino- } \\
\text { aprendizagem em Enfermagem, mostram-se } \\
\text { aliar a formação em enfermagem ao } \\
\text { ambiente virtual inova e ao mesmo tempo } \\
\text { dinamiza a forma de ensinar. }\end{array}$ \\
\hline P5 & $\mid \begin{array}{ll}\text { Uso } & \text { de } \\
\text { Moodle } & \text { na } \\
\text { disciplina } & \text { de } \\
\text { informátic } & \text { a } \\
\text { em } & \text { enfermag } \\
\text { em } & \end{array}$ & $\begin{array}{l}\text { SALVADO R, } \\
\text { M.E.; } \\
\text { SAKUMOT O, } \\
\text { M.; MARIN, } \\
\text { H.F., 2013. }\end{array}$ & $\begin{array}{l}\text { Estudo } \\
\text { transversal } \\
\text { com utilização } \\
\text { de Moodle. }\end{array}$ & $\begin{array}{l}\text { O Moodle mostrou-se um método } \\
\text { complementar ao ensino presencial, } \\
\text { considerando-se o desempenho dos alunos. } \\
\text { Introduzir o conhecimento da Tecnologia da } \\
\text { Informação durante a graduação pode auxiliar } \\
\text { na formação de futuros enfermeiros, capazes } \\
\text { de produzir serviços e gerenciar o cuidado } \\
\text { seguro e eficaz. Por meio de novas } \\
\text { habilidades e competências tecnológicas, } \\
\text { estes profissionais estarão aptos em resposta } \\
\text { à demanda de formação especializada para o } \\
\text { setor de saúde. }\end{array}$ \\
\hline
\end{tabular}

Fonte: elaborado pelos autores, 2019. (Dados da pesquisa) 
Uma das características apresentadas nos estudos tem sido a percepção a cerca do processo de educação permanente como prática educacional, uma vez que esta baseia-se por um processo que permite a melhora da atuação profissional e aprimoração contínua das habilidades.

No estudo de VILELA, et al. (2017), a apresentação e repercussão do cuidado em saúde são pontos destacados para que o enfermeiro em sua formação, tenha o conhecimento adequado para a implementação de estratégias e prevenção de erros, como exemplo, a correta administração de medicamentos. Sendo assim a educação permanente é uma estratégia contributiva para o trabalho do enfermeiro e reflete positivamente no usuário. Destarte, pode compreender que esta é uma preocupação da atual condição na formação em saúde - prevenção de danos e agravos à saúde, como forma de segurança do paciente.

Logo, destaca-se que nos estudos Tibes et al. (2017) e Freguia, et al. (2016), que o acúmulo de conhecimento é uma forma que impulsiona a educação para uma formação fragmentada, altamente especializada e com eficiência técnica-científica. Reiterando que o uso de WebQuest, por exemplo, utilizado como ferramenta de ensino, visa adquirir e integrar o conhecimento e deve ser planejado em uma a três aulas, para que o discente compreenda ao término do uso, o sentido das informações. Neste sentido reflete para uma utilização das tecnologias de forma mais consciente bem como significativa.

A partir do envolvimento do aluno, Freguia, et al. (2016), referem que o uso de WebQuest é um componente primordial, por permitir ao discente, trazer a internet e outros recursos tecnológicos para a prática pedagógica e assim potencializar o processo do ensino e aprendizagem favorecendo para construção, ampliação e manutenção do conhecimento. Contudo, é necessário que para a formação no ensino superior em Enfermagem no Brasil, na promoção de autonomia, raciocínio crítico e reflexivo é também imprescindível conteúdos de natureza atitudinal e relacional, afirmam Freguia, et al. (2016) e Leite et al. (2014).

Deste modo, é notório que o uso da tecnologia para o desenvolvimento de práticas e saberes no processo de construção de aprendizagem permite integração de modo singular diante a realidade do público alvo. Logo, frente à educação, é 
imprescindível que o domínio de ferramentas como computadores, tablets e dispositivos que auxiliem na construção e desenvolvimento de atividades. Condições esta, que no contemporâneo é disparador pela facilidade de acesso aos meios tecnológicos.

Destarte, para Leite et al. (2014), as condições causais, inerentes, intervenientes e consequências relacionadas às Tecnologias da Informação e Comunicação (TIC) no processo de ensino-aprendizagem em Enfermagem, mostram-se aliar a formação em enfermagem ao ambiente virtual inova e ao mesmo tempo dinamiza a forma de ensinar, pois amplia os recursos metodológicos e de fato promove uma maior interação entre os discentes, além de evidenciar a maior possibilidade de acesso, uma vez que reflete em uma flexibilidade e versatilidade das plataformas de ensino.

Entretanto, é importante destacar e evidenciar ainda que a introdução das tecnologias como auxiliadora do processo de formação na enfermagem tem-se maior credibilidade como uso de aproximação maior do conhecimento, entretanto não substitui a prática e vivencia do contexto real, mas pode contribuir para o acesso de informações que vão auxiliar na conduta e prática profissional. Outrossim, pode contribuir na produção de serviços e gerenciamento do cuidado seguro e eficaz, ao passo que, proporciona o surgimento de novas habilidades e competências.

Contudo, a formação e estudos em Educação à Distância (EAD) tem apresentado benefícios ao público, referenciado em Salvador, Sakumoto e Marin (2013), por modificar a forma de comunicação e interação no processo de ensinoaprendizagem em interfaces dinâmicas e atraentes, ao qual garantem comunicação eficaz, desafiando a capacidade de compreensão, avaliação e reflexão do discente em horário não escolar. Porém, reforça que ampliar o acesso à informação integrando diversificados métodos não prudente, caso não ocorra o comprometimento e, sobretudo, a motivação do corpo docente.

Neste aspecto mostra que deve-se ter a responsabilização e envolvimento do discente acerca do uso das tecnologias de ensino, em especial quando se refere no processo aprendizagem e construção do conhecimento, pois este não só evidencia a flexibilidade do acesso ao ensino, mas requer participação efetiva do indivíduo. Outro ponto importante é que o educador também possa reconhecer esse 
instrumento como forma metodológica eficiente e a partir disso motiva-se e promover o processo educacional significativo.

Logo, o estudo evidencia que o impacto das Tecnologias da Informação e Comunicação (TICs) nas instituições de ensino superior - aspecto este cabível de se destacar que o uso ferramentas do tipo educação à distância, tem sido aliado ao processo de educação continuada de profissionais já graduados conforme apresentam os estudos selecionados a partir do cruzamento de dados para o elencamento desta pesquisa.

\section{CONCLUSÃO}

Observa-se nos estudos um amplo aspecto acerca dos benefícios e riscos do ensino em enfermagem por meio do uso de plataformas e interação colaborativa por WebQuest suprindo lacunas na formação. Dentre as quais, se destaca o processo de promoção à saúde na formação continuada para a prevenção de erros, bem como a decisão do uso desta metodologia de ensino, contribui para a melhoria dos serviços de saúde.

Contudo, destaca-se que o melhor comportamento para este tipo de formação está atrelado a educação permanente e continuada dos profissionais, e em nível de especialização, aprimoramento e aperfeiçoamento profissional. Tais condições refletem pela flexibilidade de organização do cronograma de estudos, contribuição e interação na comunicação e organização dos trabalhos em meio virtual e pelo caráter de acumulo de conhecimento pela facilidade de acesso a conteúdos durante a participação dos momentos de estudos.

Frente a estas condições, ressalta-se que ainda existem lacunas nos estudos acerca da evidência sobre a vivência de estudantes á nível de graduação sobre o uso das tecnologias. Sendo assim, este trabalho um disparador para a formulação de estudos e hipóteses de implementação de ferramentas eficazes para a produção do conhecimento e formação de novos profissionais na área da enfermagem. 


\section{REFERÊNCIAS BIBLIOGRÁFICAS}

ARAUJO, A.S. et al. Atuação do enfermeiro na assistência ao pré-natal versus sífilis: uma revisão integrativa. Interfaces Científicas - Saúde e Ambiente, Aracaju, 2018, v.6, n.2, p. 95110. Disponível em:< https://periodicos.set.edu.br/index.php/saude/article/view/4626/pdf $>$. Acesso em: 01 de out. de 2018.

BRASIL, 1996. Lei n. 9.394, de dezembro de 1996. Estalece as diretrizes e bases da educação nacional. Lei de Diretrizes e Bases da Educação - LDB. [internet] 1996. Disponível em: < https://presrepublica.jusbrasil.com.br/legislacao/109224/lei-de-diretrizes-e-bases-lei-9394- 96>. Acesso em: 24 de nov. de 2018.

FREGUIA, A.F.S. et al. Acidente de trabalho como material biológico no contexto de um ambiente virtual de aprendizagem. CuidArte Enfermegem, 2016 Jul-dez.; v. 10, n. 2, p. 102108. Disponível em: < http://www.webfipa.net/facfipa/ner/sumarios/cuidarte/2016v2/102108.pdf $>$. Acesso em 24 de nov. de 2018.

LEITE, K.N.S. Vivenciando o uso das Tecnologias da Informação e Comunicação e suas dificuldades no processo ensino-aprendizagem. Dissertação (Mestrado) - UFPB/CCS, João Pessoa, 2014. Disponível em:

$<$ https://repositorio.ufpb.br/jspui/bitstream/tede/5151/1/arquivototal.pdf>. Acesso em: 24 de nov. de 2018.

MOREIRA, A.F.; JUNIOR, P.M.S. Conhecimento escolar nos currículos das escolas públicas: reflexões e apostas. Currículo sem Fronteiras, v.17, n.3, p.489-500, set./dez. 2017. Disponível em: http://www.curriculosemfronteiras.org/vol17iss3articles/moreira-silva.pdf. Acesso em: 01 de out. de 2018.

PRADO, C. et al. Ambiente virtual de aprendizagem no ensino de enfermagem: relato de experiência. Rev. Bras. Enferm. [internet] 2012 Set., v.65, n.5, p.5, Disponível em: < http://www.scielo.br/scielo.php?script=sci_arttext\&pid=S0034-71672012000500022>. Acesso em 24 de nov. de 2018.

SALVADOR, M.E.; SAKUMOTO, M.; MARIN, H.F. Uso do Moodle na Disciplina de Informática em Enfermagem. J Healt Inform., 2013, out-dez., v. 5, n. 4, p. 121-126. Disponível em: <http://www.jhi-sbis.saude.ws/ojs-jhi/index.php/jhi- sbis/article/view/268/181>. Acesso em: 24 de nov. de 2018.

TIBES, C.M. et al. Desenvolvimento de recursos educacionais digitais para o ensino em enfermagem. Rev. enferm UFPE on-line., Recife,2017, v.11 (Supl. 3), p. 1326-1334. Disponível em: <https://periodicos.ufpe.br/revistas/revistaenfermagem/article/view/13972/16815>. Acesso em 24 de nov. de 2018.

VILELA, R.P.B. et al. Educação Permanente: Tecnologia para a prevenção do erro de medicação. CuidArte Enfermegem, 2017 Jul-dez.; v. 11, n. 2, p. 203-208. Disponível em: < http://www.webfipa.net/facfipa/ner/sumarios/cuidarte/2017v2/203.pdf $>$. Acesso em 23 de nov. de 2018. 